\title{
Research Gaps and Opportunities in Sensor-Based Medical Exploration Capabilities in Extravehicular Astronaut Suits
}

Matthew Morrison ${ }^{1 *}$, Rodrick Rogers ${ }^{1}$, Karanvir Singh ${ }^{1}$, Bryan Harper ${ }^{1}$, Francisco Sanchez ${ }^{1}$, Olivia Williams $^{1}$, Travis Williams ${ }^{1}$, Ryan Mays ${ }^{1}$, Chenye Li ${ }^{1}$, Jazsmin Arnold ${ }^{1}$, Georgia Haggard ${ }^{1}$, Hana Smith ${ }^{1}$, Erica Sims ${ }^{1}$, Lauren Woodard Parrish ${ }^{2}$, Melinda Valliant ${ }^{2}$ and John Ralston ${ }^{3}$

${ }^{1}$ Department of Electrical Engineering, University of Mississippi, Oxford, Mississippi, USA

${ }^{2}$ Department of Nutrition and Hospitality Management, University of Mississippi, Oxford, Mississippi, USA

${ }^{3}$ Protxx Inc., Menlo Park, California, USA

\begin{abstract}
NASA's Human Research Program has identified the need to improve their capability to predict estimated medical risks during exploration missions, as well as the need to provide computed medical decision support while minimizing medical resource utilization. From May 2017 through October 2017, a research team with the University of Mississippi Electrical Engineering Department conducted interviews with a dozen NASA personnel from the Human Research Program, Life Support Systems Technology Development, Game Changing Development Program, the Wyle Science, Technology and Engineering Group, and MEI Technologies. This report outlines the challenges and gaps identified as a result of these interviews in the Human Research Roadmap towards implementing a sensor-based medical monitoring system in EVA suits, as well as the opportunities in pursuing solutions towards these challenges. We describe the knowledge gaps in determining a clear definition for which measurable EVA suit environment and astronaut medical conditions are mission critical. We detail the current principle and special challenges of monitoring mission critical measurables in micro-gravity and zero-gravity environments with respect to their sensing ability. We then propose a three-stage research framework for meeting these objectives that is robust in scope, yet partitioned such that innovations or setbacks in one stage will not unduly harm progress. First, identifying a set mission critical measurable will enable innovations in sensor networks in EVA suits. We discuss proposed approaches to monitoring astronaut health and environments and relevant gaps. The second stage is using data from the sensor network as inputs to algorithms for determining when mission-critical parameters have been violated, as well as priorities for reporting that information. The third research area focuses on secure and reliable delivery of sensor information to the IV crewmembers, along with rendering of mission-critical information in a Heads-Up Display (HUD) worn by the astronaut. We discuss the current status of HUD technology in EVA suits and the challenges towards advancing that technology for mission deployment. We also discuss challenges in how the astronaut and IV crewmembers will utilize logged health and tracking information operationally. Finally, the status of the work already conducted under the proposed research framework is discussed.
\end{abstract}

Keywords: Beyond Low Earth Orbit; Extravehicular Astronaut Suit; Heads-Up Display; Mission Critical Measurables

Abbreviations: BLEO: Beyond Low Earth Orbit; CHSP: Center for Health and Sports Performance; COTS: Commercial Off the Shelf; EEMO: Extreme Environment Mission Operations; ECG: Electrocardiography; EE: Electrical Engineering; ELS: Exploration Life Support; EPSP: EVA Physiology, Systems, and Performance; EVA: Extravehicular Activity; ExMC: Exploration Medical System; HITG: Heads in the Game; HRP: Human Research Program; HUD: HeadsUp Display; IDEAS: Integrated Display and Environmental Awareness System; IVA: Intravehicular Activity; ISS: International Space Station; MCM: Mission Critical Measurable; MEMS: Microelectromechanical System; OLED: Organic Light Emitting Diode; PLSS: Portable Life Support System; RCA: Rapid Cycle Amine; UM: University of Mississippi; UMMC: University of Mississippi Medical Center; VOR: Variable Oxygen Regulator

\section{Introduction}

This report is the result of a six-month study conducted by the University of Mississippi Electrical Engineering (UM-EE) Department in collaboration with Protxx, Inc. and the UM Center for Health and Sports Performance (UM-CHSP), and presents high-level findings and recommendations regarding the direction of sensor-based medical exploration capabilities in extravehicular (EVA) suits. NASA's Human Exploration and Operations Mission Directorate and the Human Research Program (HRP) provide solutions to critical problems that place human exploration missions and their crews at risk. In order to successfully conduct space exploration missions beyond low-Earth orbit (BLEO), potentially transformative research in technologies and countermeasures against risks to crew health and performance [1] must be investigated. EVA lunar and Martian missions are planned to last 30 times longer than the Apollo missions, and a collaborative effort is required to determine which objective medical data must be collected and monitored to ensure successful missions in microgravity environments.

During our study, we conducted interviews with the following NASA personnel: From NASA HRP: Dr. John Charles and Dr. Jennifer Fogarty; from Wyle Labs: Dr. Lauren Merkle, Dr. Sara Zwart, Mr. Nathaniel Newby, Mr. David Ham, Dr. Jeffrey Somers, and Dr. Shane McFarland; Dr. Daniel Barta from Life Support Systems Technology

*Corresponding author: Matthew Morrison, Department of Electrical Engineering University of Mississippi, Oxford, Mississippi, USA, Tel: 662-915-5384; E-mail: morrison@olemiss.edu

Received November 06, 2017; Accepted November 20, 2017; Published November 27, 2017

Citation: Morrison M, Rogers R, Singh K, Harper B, Sanchez F, et al. (2017) Research Gaps and Opportunities in Sensor-Based Medical Exploration Capabilities in Extravehicular Astronaut Suits. J Biosens Bioelectron 8: 248. doi: 10.4172/2155-6210.1000248

Copyright: (c) 2017 Morrison M, et al. This is an open-access article distributed under the terms of the Creative Commons Attribution License, which permits unrestricted use, distribution, and reproduction in any medium, provided the original author and source are credited. 
Development; Mr. David Miranda from the Integrated Display and Environmental Awareness System project; Dr. Andrew Abercromby from Extreme Environment Mission Operations (EEMO); and Mr. William Parsons, former director of Kennedy and Stennis Space Centers. These NASA professionals range in expertise from clinical translation, cardiovascular discipline scientist, human system risk management, biomedical research and environmental sciences, nutritional biochemistry, space exercise wearables, space medicine advanced projects, astronaut occupant protection, spacesuit mobility, exploration life support systems, head-mounted displays, neurosciences, anthropometry, and biomechanics.

We then conducted interviews with astronauts to gain expertise in the current standards for astronaut training and application of EVA communications. The three interviewees were Dr. Igor Dubinsky, a current finalist in the NASA Astronaut Selection Process, Dr. Nicole Stott, a veteran of four space shuttle missions as well as one International Space Station mission. Finally, to learn about BLEO environments for the suits, we interviewed General Charles Duke, who served as CAPCOM for Apollo 11 and performed 20 hours of EVA operations on the Moon during Apollo 16. As a result of these interviews, we have identified a set of research gaps that must be addressed in order to ensure successful monitoring of crew health and performance beyond LEO. Specifically, there are three major areas that require further investigation.

First, in order to mitigate significant risks to astronauts, there must be a coordinated effort to determine a set and hierarchy of missioncritical measurables (MCM) of medical, environmental and human factors that require real-time monitoring during EVA missions. The lack of a clear consensus is a major barrier to defining and prioritizing physiological and EVA system parameters that must be displayed. There exists a roadmap gap, EVA 10 [2], which addresses real-time physiological and system parameters, but the determination of factors does not account for limits in technology for monitoring and displaying that information to the astronaut or to intravehicular (IVA) crew members. Establishing a hierarchy for transmitting and displaying that information will be essential to implementing any bioadvisory algorithms.

Second, research must be conducted into biosensing and biodiversity devices and algorithms for real-time sensors and feedback systems in autonomous EVA suits. NASA's ability to predict and estimate medical risks BLEO is complicated by the fact that medical risk to the crew increases as medical assets are consumed during a mission [3]. The medical system must be optimized for mass, volume, power consumption, and data transmission while simultaneously optimizing logged health and tracking information to benefit the crew during the mission. Device testing must ensure long-term reliability because of the limited ability to resupply and troubleshoot these devices BLEO, especially when device failure could result in aborted missions. Determining the corresponding MCMs will assist in optimizing the devices and reduce the time to close roadmap gaps. We must also identify and address the unique challenges of monitoring MCMs using biosensors in micro- and zero-gravity environments, including power consumption, durability, radiation, and gravity.

Third, we must develop information transmission and display systems that meet the needs and challenges of EVA personnel and missions. Algorithms for determining autonomous and augmented medical decision support must be integrated into the exploration medical system (ExMC) by optimizing crew interfacing with medical decision support and accounting for telemedicine limitations [4]. Information that must be immediately conveyed to the EVA astronaut must be displayed in a non-invasive manner that does not compromise the ability of the astronaut to adjust to the needs of the mission or obfuscate their vision. Data that must be transmitted to IVA astronauts must be reliably and accurately transmitted in a concise manner that allows for rapid and accurate decisions. By determining an appropriate set of mission-critical measurables, the amount of relevant ExMC sensor data to be transmitted and displayed can be minimized, preventing "information fatigue", reducing complexity, and improving reliability.

In this report, we will detail the feedback received from NASA personnel, and relate the relevant research challenges to the currently existing Human Research Roadmap. Next, we propose a high-level plan for meeting those research challenges that is partitioned by the three major challenges described above. We discuss the infrastructure at UM$\mathrm{EE}$ and UM-CHSP and the avenues for collaboration on these topics.

\section{Relevant Research Challenges}

\section{Astronaut health monitoring: From ISS to BLEO}

Most evaluations of astronaut health, nutrition, physical fitness, and psychological behavior involve pre- and post-spaceflight assessments [5]. While certain ergometer aerobic capacity inflight data are monitored and collected, significant issues remain in obtaining the biodosimetry and dietary intake data required for accurate assessments of the impact of spaceflight on an astronaut's bone densitometry, body mass, strength and conditioning, blood chemistry, and psychological health. The International Space Station (ISS) currently contains medical subsystems including: the Countermeasures System (CSS), which monitors the impact of living in microgravity; the Environmental Health System (EHS), which checks for contaminants in the ISS atmosphere; and the Health Maintenance System (HMS), which includes medical packs, life support systems, and physiological monitoring software.

However, multiple NASA experts indicated significant issues in monitoring astronaut health that must be addressed in order to properly determine which measures compromise "mission critical." We were told that the medical monitoring technological capability on the ISS is less than that found in a conventional ambulance. After interviewing nutrition experts and ISS technical advisors, we determined a clear need for advances in medical monitoring capabilities on ISS with a focus on deployment for BLEO missions and enabling astronauts to participate in these monitoring techniques.

According to Dr. Sara Zwart, astronauts currently use the Philips Actiwatch Spectrum System [6,7], which utilizes accelerometer-based measurements of an astronaut's motionto monitor circadian rhythms, sleep-wake patterns, and other sleep issues, such as hyperactivity and other daily routines. The primary issue with sleep monitoring is that approximately $15 \%$ of astronauts adapting to microgravity develop Space Adaption Back Pain (SABP) [8] due to spinal lengthening. Mr. Nathaniel Newby stated that "monitoring is vital to help with the prevention of broken teeth and back injuries due to bone and muscle deconditioning." Use of Actiwatch permits direct correlation of realtime sleep data to incidences of SABP, allowing NASA to fill the research gap with reliable data. However, most health care measurements of astronauts are limited to pre- and post-flight. For example, during missions, gravity and muscles are no longer able to work together to evenly distribute fluids inside the body. Without gravity, bodily fluids often shift towards the head and chest. The lack of astronaut hydration monitoring can increase the risk of many health-related complications. Additionally, bone density and Vitamin D testing is only collected preand post-flight. 
Further exacerbating the problem is the issue of documenting astronaut dietary intake. Food is currently stored in a central container, and is not individually labeled. Once a week, crewmembers are asked to complete a food frequency questionnaire, but astronauts often fall short of the task. The lack of monitoring gives the astronauts behavioral freedom that can lead to dietary deficiencies and regressions in physical performance. Current methods for astronauts to record food intake include NASA's database search, appendix search, search by picture, and barcode search. Attempts to use bar-coded scanners on computer devices were met with resistance from ISS crewmembers due to difficulty of scanning, especially if the food made the wrapper "bumpy" and difficult to scan. Any alternative solution to monitoring nutrition on spaceflights, such as using RFID tags, must be intuitive and reliable, while also being designed to motivate and encourage astronauts to use the solution.

\section{EVA Suit monitoring challenges}

The current EVA suit is designed for short-term missions with durations up to about 6-8 h. While over 300 EVA missions have been performed, only 14 lunar EVA missions were ever conducted, meaning that we must plan for EVA missions that may last up to $24 \mathrm{~h}$ BLEO while accounting for systems and protocols that maximize human performance and reduce safety risks with minimal understanding of long-term effects of microgravity on the human body. The EVA Physiology, Systems, and Performance (EPSP) Project is working to coordinate these efforts, but face significant challenges. The Apollo astronauts recommended increased functional capability through increased suit flexibility, lower center-of-gravity, lower internal pressure, and increased ambulatory and medical monitoring $[9,10]$. Additionally, astronauts have consistently expressed frustration with the time consuming and cumbersome procedures required to put on, use, and remove current biomedical sensor systems.

Since no food or water is consumed during an EVA mission, there is currently no understanding of how EVA missions affect an astronaut's hydration level and other important physiological parameters. These factors must be investigated to better quantify the impacts of Decompression Sickness (DCS) [9], which is especially important since EVA missions are known to require significant strain and effort. The Apollo astronauts described ambulating on the lunar surface as more akin to moving on the surface of an ocean as opposed to walking through a desert [11], requiring significantly more effort from their quadriceps muscle. Additionally, they described only $10 \%$ efficiency in the use of their hands, as well as fingernail and hand trauma [12], resulting from excessive strain and effort. Quantifying these additional physiological issues before and during spaceflight, in order to determine appropriate MCMs, is essential for successful BLEO EVA missions.

\section{Heads-Up Displays (HUD) potential and challenges}

During our interviews, we determined the need to convey information to EVA astronauts and IVA crewmembers through a HUD. We determined during our interview with Mr. David Ham that we must consider the physiological and physical strain the HUD will have on the astronauts. We must be able to display information to the astronaut that is simple to discern and easy to act upon without compromising the astronaut's ability to complete their mission [13,14]. Any HUD display must take into account vertical and horizontal boresight offsets, accurate presentation of information, customization of information for each astronaut, as well as the need to minimize technical complexity while providing a level of utility high enough to motivate and encourage use of the HUD. Previous studies have shown that mean response times to HUD information in military environments was longer than to direct stimuli ( 4.13 vs. $1.75 \mathrm{sec}$ ) [15]. Therefore, any design of a HUD display must consider which information is essential to the astronaut in order to minimize the stimulus, reduce information overload, and ensure appropriate response times. Additionally, during EVA missions, the HUD must not obfuscate the vision of the astronaut, either during display or in the event the HUD breaks down, in order to avoid compromising the mission or placing the astronaut at risk.

The NASA Integrated Display and Environmental Awareness System (IDEAS) project [16] conducted under Mr. David Miranda at Kennedy Space Center under the Space Technology Mission Directorate is working to develop optical head-mounted displays in order to have a transparent, interactive display that can improve the astronaut's situational awareness by combining augmented reality and visual communication [17]. According to Mr. Miranda, the focus of this project has been on gas sensors and nanosensors made by NASA at AMES, with relatively little focus on displaying data from biosensors. The IDEAS system includes capabilities for astronauts to chat and see each other working on tasks, while simultaneously displaying gas levels in the work environment. The framework uses commercial-off-theshelf (COTS) smart glasses with android-enabled IDEAS software.

We identified several challenges in integrating a Heads-Up Display into the EVA suit. First, if a color-coded system is put into play, color blind people may not properly interpret some warnings; this issue via an adaptive display that can also present single-color numbers, symbols, or other graphical representations to display that there is a problem. Second, astronauts may not want to use the HUD due to the potential for motion sickness. A common refrain we heard from several experts is "astronauts talk", and if there is a bad experience with the HUD, then the astronauts would tell other astronauts not to use the HUD. Additionally, multiple NASA personnel liked our suggestion for a library and menu of services with which the astronauts could personalize the information on the HUD prior to each EVA mission, since each astronaut has different missions and jobs that may each require different information and alerts displayed $[18,19]$.

\section{Life support system technologies}

The current EVA life support system contains duration limiting hardware, and their limited flexibility presents additional challenges for their use into BLEO missions for Exploration Life Support (ELS). According to Dr. Daniel Barta, the current state of the EVA Portable Life Support System (PLSS) consists of two parts [20]:

- A Variable Oxygen Regulator (VOR), which controls the nominal pressure in the space suit, and provides 8,000 pressure set points to increase flexibility and safety, and permit robust integration of sensors across future generations of spacecraft and missions; and

- A Rapid Cycle Amine (RCA) swing bed, which is a vacuumswing based adsorption intake system which sucks at the wrist and ankles and blows at the head to remove $\mathrm{CO}_{2}$ from a flowing ventilation garment that consists of long underwear with integrated cooling tubes. The objective is a dual-function system that also removes any humidity around the mouth and chest areas that might cause condensation.

The EVA atmosphere is $\mathrm{O}_{2}$, which makes fires a concern in the presence of batteries and electronics. $\mathrm{CO}_{2}$ and $\mathrm{O}_{2}$ concentrations, humidity, and temperature would be beneficial measurements to minimize degradation of electronics. The EVA backpack has limited power, is not pressurized, is exposed to extreme hot and cold temperatures, and contains only well-insulated devices that rely on 
convection for heat removal. The space suit pressure garment requires innovative technologies that increase the life, comfort, mobility, and durability of gloves, self-sealing materials to minimize the effects of small punctures or tears, and materials that are resistant to abrasion $[21,22]$. Focused research is needed for a PLSS that can measure critical life support constituents in a single combined flow-through sensor configuration. Free water tolerance is another important feature.

\section{Extreme environment mission operations}

A major challenge in determining which MCMs must be monitored in the EVA suit is determining how humans react in BLEO environments. To this point, there is little research in evaluating the metabolic cost to the EVA astronaut's mobility [23]. Additionally, there is a clear challenge in mitigating information overload to the astronaut in Extreme Environment Mission Operations (EEMO). According to Dr. Andrew Abercromby, there has been some work with NASA and UCI regarding how much information should be displayed, including using color slides and guides. There is a clear need to be able to display information to the astronaut in a manner that allows them to clearly determine when there is an issue even before they know the extent of the issue. Additionally, we still have a knowledge gap with respect to having a clear definition of what is critical and what is enhancing information for EVA in BLEO environments.

\section{Astronaut Feedback}

In the next stage of our research, we conducted interviews with three astronauts to gain expertise in current standards for astronaut training, EVA missions on the ISS, and EVA missions in BELO environments. To gain expertise as to how NASA's astronaut training program incorporates sensors to improve their capabilities, we interviewed Dr. Igor Dubinsky, who is involved in the NASA Astronaut Selection Process (and was referred to us by Mr. Bill Parsons, the former director of the NASA Kennedy Space Center). Dr. Dubinsky emphasized his desire for a Heads-Up Display, but indicated that most HUD technologies have information overload. Second, Dr. Nicole Stott, who has served on four shuttle and ISS missions, indicated that she would specifically like to have the option to view on a HUD the checklists that IVA personnel currently send to a display on the cuff outside the suit, because of the difficulty in accessing the information, as well as the ability of the EVA and IVA to turn off the HUD display for redundancy and safety. Dr. Stott further indicated that a HUD would be especially helpful in showing an EVA participant the appropriate translation path to get back to the station. Third, we interviewed General Charles Duke, who is a veteran of Apollo 16. He said that the Apollo astronauts had practiced all activities hundreds of times on Earth, but if a mission were to exceed three days, or if any improvisation was required, then a HUD would be required to update the mission.

\section{Biosensors in Space: Principle and Special Challenges}

NASA has identified the need to develop the capability to comprehensively monitor and process medically-relevant information to support medical operations, and enhance operational innovation for astronauts' medical needs by implementing biosensors in their Exploration Medical System [23]. Within this task, they aim to certify a single Electrocardiography (ECG) system that can perform both 12 lead diagnostic and reduced lead ambulatory measurements, as well as identify dry electrodes to replace commonly used wet electrodes during EVA missions. The astronauts themselves are averse to excessive use of biomedical devices during flight. For example, most early astronauts in the Mercury mission wore ECGs, breathing-detecting thermistors, and a rectal probe for accurate core body temperature measurement [24]. The engineers found that the thermistor did not accurately measure respiration, and eventually evolved to an impedance pneumograph, which leverages thoracic electrodes to determine the respiratory rate and volume [25]. Studies have been conducted in the use of sensors for muscle oxygen and $\mathrm{pH}$ during walking and running in simulated microgravity [26]. Excessive use of probes and medical sensors may limit the mobility of EVA personnel, especially as the missions extend to 24 hours.

NASA also identifies and evaluates COTS devices for health monitoring in space environments. Any biomedical device to be used in spaceflight must undergo a rigorous testing, certification, and delivery program at the International Space Station Medical Projects center to protect the health and performance of crewmembers. For example, any sensor affixed to the astronaut's head would specifically be tested for compatibility to the Sokol snoopy cap, to ensure that excessive noise or interference are not introduced by the sensor or its electronic interface. While IoT devices designed for Earth environments are designed for long battery lifetime, such biosensor devices used in space will likely need to harvest energy from the surrounding environment and be able to operate on nanowatt power budgets [27].

However, the use of small form-factor biosensors may serve as a potential advantage in mitigating the weight of the space flight vehicle. For example, brain imaging for spaceflight is a unique challenge, because clinical neuroimaging tools are too large, heavy, and powerdemanding for spaceflight. NASA has also specifically identified the need to better understand the risk of injury associated with crewed vehicle landings and how this risk relates to the desired acceptable risk. Head injury is currently listed as "not addressed" on NASA's Medical Conditions List. Correlating affixed COTS sensor data with highangular-resolution diffusion spectrum MRI (DSI) imaging, including multidimensional diffusion anisotropy characterization of neural tissue damage, may provide a promising neuromechanical biomarker [28] for real-time monitoring and alerting of cumulative head impact loading and injury risks in astronauts.

We describe below two particular challenges in implementing low-power biosensors with small form factors in space environments. First, we discuss the impact of micro- and zero-gravity on the sensing capabilities of the devices. Next, we describe the current state of energy harvesting in these environments.

\section{Medical sensors in micro- and zero-gravity environments}

Microelectromechanical systems (MEMS) sensors integrate highly sensitive accelerometers and gyroscopes as inertial measurement units. Microgravity environments are subject to quasi-steady accelerations, higher frequency acceleration, and transient disturbances [29]. Assumptions that engineers may validly make, such as near-constant gravity of $9.8 \mathrm{~m} / \mathrm{s}^{2}$, relatively easy access to power outlets for recharging, form factor and weight requirements, and access to replacement parts are no longer valid for biosensors in BLEO environments. Space flight planning also requires significant considerations of payload weight, which means traveling with many backup medical devices will be discouraged. The sensors must also support contingency operations, such as delayed landings, by providing sufficient battery time, recording capability, triggering, and access to raw data for subsequent analysis.

MEMS accelerometers and gyroscopes typically incorporate a micromachined proof mass, which is connected to one or more capacitive sensing elements. Linear and rotational motion of the proof 
mass are translated to the capacitance elements, and the resulting changes in capacitance are proportional to the linear acceleration (in the case of a MEMS accelerometer) or the angular velocity (in the case of a MEMS gyroscope). MEMS accelerometers and gyroscopes commonly used in biosensors typically compensate for gravitational acceleration. Compensation algorithms expecting a gravitational force of approximately $9.8 \mathrm{~m} / \mathrm{s}^{2}$ for Earth's gravity would produce inaccurate results on the Moon and Mars, where the force due to gravity is 1.622 $\mathrm{m} / \mathrm{s}^{2}$ and $3.711 \mathrm{~m} / \mathrm{s}^{2}$, respectively. The microgravity conditions on the International Space Station that result from its rapid orbit around the Earth are relatively constant. Significant errors in MEMS gyroscope readings can also be caused by their sensitivity to vibrations, which is strongly dependent on the frequency and magnitude of the vibrations, and can require complex compensation schemes.

The previously mentioned Actiwatch Spectrum System uses a MEMS accelerometer and color sensitive photodiodes to monitor and analyze movement during circadian rhythms [30]. Protxxhead impact dosimeters incorporate MEMS accelerometers and gyroscopes to monitor linear and rotational head impact motion and resulting deformations of neural tissues. The mechanical impact exposure can then be correlated with other athlete (or astronaut) performance assessments and clinical data to determine a biomarker for brain wellness [31]. The Actiwatch samples circadian rhythm data at a rate of $32 \mathrm{~Hz}$, while the Protxx sensor can adapt to a wide range of biomechanics monitoring requirements by sampling sample motion data between $10 \mathrm{~Hz}$ and 3 $\mathrm{kHz}$, for all six degrees of freedom (3 linear axes, 3 rotational axes) [32]. The power consumption of a computing device is directly proportional to the operating frequency, where $C_{L}$ is the load capacitance, $V_{d d}$ is the supply voltage, and $\mathrm{f}$ is the operating frequency: $\mathrm{P}=1 /{ }_{2} \mathrm{C}_{\mathrm{L}} \mathrm{V}_{\mathrm{dd}}{ }^{2} \mathrm{f}$. This presents a special challenge, as the accuracy of measurement increases as the sampling rate increases. Therefore, the accuracy of any medical sensing device is inversely proportional to its power consumption. Careful design consideration for any medical device implemented in BLEO environments must consider both the tradeoffs in measurement accuracy, sampling frequency, and power consumption.

An astronaut on a BLEO mission may go through many changes in gravity and vehicle acceleration/deceleration. The gravitational forces experienced by the astronaut may change from normal Earth gravity to the virtually zero-gravity environment between Earth and the destination planet, and then the lower-gravity environment that would be encountered at destination such as the Moon or Mars. Therefore, any medical sensor which utilizes an accelerometer must incorporate adaptive algorithms to compensate for the current gravity and vehicle acceleration experienced by the astronaut.

\section{Energy harvesting in space environments}

Energy harvesting is a technique where energy is drawn from the environment immediately external of the device. Research is being conducted into the use of heat, light, movement, and vibrations to scavenge power for a device to minimize or eliminate reliance on batteries. Energy harvesting is a promising approach to reducing the size and power requirements of medical sensors, and could facilitate the use of inaccessible sensors in astronaut suits for long-duration EVA missions.

Current approaches include harvesting of ambient radio frequency energy [33] and photovoltaic power generation [34]. The most promising energy harvesting technique for medical sensors in BLEO environments is the use of piezoelectricity [35]. Piezoelectric materials generate small voltages upon deformation, allowing mechanical motion and vibrations to be exploited for piezoelectric charging. NASA has specifically combined polyvinylidene fluoride with carbon electrodes to improve conductance and durability, targeting applications in wearable electronics, as well as wind and mechanical energy harvesting [36].

Using piezoelectric approaches also has the potential to improve the sensing capability of a medical biosensor. Some MEMS sensors support an ultra-low power "sleep" mode that leverages the initial movement of the proof mass to enable a wake-up function. The drawback is that any initial motion after a long sleep time is used to start up the device, and the corresponding motion data become lost, reducing the accuracy of that measurement. Adding a piezoelectric element to the design could help to shorten the turn-on time, while also allowing power generation from sensor motion and decreasing battery requirements.

\section{Research Challenges and Objectives}

Below, in Tables 1-3, we have outlined a set of Research Objectives and Challenges that need to be met to provide a safe and reliable PLSS for EVA BLEO missions. Each table lists specific research stages, the

\begin{tabular}{|c|c|c|c|}
\hline $\begin{array}{l}\text { Research } \\
\text { Stage }\end{array}$ & $\begin{array}{l}\text { Roadmap } \\
\text { Gaps }\end{array}$ & $\begin{array}{l}\text { Specific Needs } \\
\text { Addressed }\end{array}$ & Objectives and Challenges \\
\hline \multirow[t]{2}{*}{$\begin{array}{l}\text { 1.1 Exploration Mission Medical } \\
\text { Care Operations }\end{array}$} & \multirow[t]{2}{*}{$\begin{array}{l}\text { Med01, N3.1, } \\
\text { N3.2. }\end{array}$} & $\begin{array}{l}\text { Must define the ExMC } \\
\text { requirements }\end{array}$ & $\begin{array}{l}\text { - 1.1.1 Must define and determine the required level of medical monitoring and response } \\
\text { for a } 24 \text {-hour long EVA mission. } \\
\text { - 1.1.2 Must determine the MCMs both in the EVA suit and in BLEO environments before } \\
\text { we can minimize opportunity for medical errors. } \\
\text { - 1.1.3 Must determine the difference between measurables that are MCMs and are } \\
\text { "nice to have", and differentiate between those measurables in BLEO environments as } \\
\text { opposed to on the ISS. }\end{array}$ \\
\hline & & Portable Medical Care & $\begin{array}{l}\text { - 1.1.4 Must determine the number, functions, and performance requirements of medical } \\
\text { sensors that an astronaut may carry on an EVA mission on the PLSS, and if they create } \\
\text { additional risks to the EVA astronaut. }\end{array}$ \\
\hline \multirow[t]{2}{*}{$\begin{array}{l}\text { 1.2 Assessment of medical } \\
\text { issues of EVA personnel in } \\
\text { BLEO environments. }\end{array}$} & Med01 & EVA astronaut information & $\begin{array}{l}\text { - 1.2.1 Need to determine whether procedures with imagery are sufficient for the EVA } \\
\text { astronaut to autonomously determine the risks involved in continuing the mission while an } \\
\text { MCM parameter is violated. } \\
\text { - 1.2.2 Need to determine which MCMs and mission-specific messages astronauts may } \\
\text { personalize in their EVA displays for ease of use on each mission. } \\
\text { - 1.2.3 Need to determine methods for rendering graphics that are easy to use and can } \\
\text { adapt to the unique needs of color-blind EVA personnel in BLEO environments. }\end{array}$ \\
\hline & Med07 & IVA astronaut information & $\begin{array}{l}\text { - 1.2.4 Need to determine whether IVA personnel may autonomously assess an EVA } \\
\text { astronaut in the event of a medical issue. } \\
\text { - 1.2.5 Need to determine which EVA and BLEO environmental measurables are sufficient } \\
\text { for IVA personnel to assess the condition of EVA personnel. }\end{array}$ \\
\hline
\end{tabular}

Table 1: Research Objectives and Challenges: Determination of Mission-Critical Measurables 
Citation: Morrison M, Rogers R, Singh K, Harper B, Sanchez F, et al. (2017) Research Gaps and Opportunities in Sensor-Based Medical Exploration Capabilities in Extravehicular Astronaut Suits. J Biosens Bioelectron 8: 248. doi: 10.4172/2155-6210.1000248

Page 6 of 9

\begin{tabular}{|c|c|c|c|}
\hline Research Stage & Roadmap Gaps & Specific Needs Addressed & Objectives and Challenges \\
\hline \multirow[t]{2}{*}{ 2.1 EVA Sensors and Devices } & $\begin{array}{l}\text { Med08, } \\
\text { BMed2 }\end{array}$ & $\begin{array}{l}\text { Heart Rate, Blood Oxygen, } \\
\text { EKG, Hydration }\end{array}$ & $\begin{array}{l}\text { - 2.1.1 Must determine the extent that dehydration has on astronaut performance during } \\
\text { EVA operations in real time instead of pre- and post-flight evaluations. }\end{array}$ \\
\hline & $\begin{array}{l}\text { Med09, } \\
\text { BMed7 }\end{array}$ & Environmental Monitors & $\begin{array}{l}\text { - 2.1.2 Must determine which environmental variables must be measured to contribute } \\
\text { to EVA personnel's BLEO situational awareness, and design those sensors. } \\
\text { - 2.1.3 Must determine challenges with integration of the sensors into the EVA suit to } \\
\text { accurately measure the environment without compromising EVA suit readiness. }\end{array}$ \\
\hline \multirow[t]{2}{*}{$\begin{array}{l}\text { 2.2 Reduction of Sensor } \\
\text { Resources in EVA Suit }\end{array}$} & Med01 & Minimal Required Sensors & $\begin{array}{l}\text { - 2.2.1 Must determine which sensor measurables contribute to the baseline for EVA } \\
\text { missions. } \\
\text { - 2.2.2 Cannot accomplish until the ExMC requirements are known, and their potential } \\
\text { for integrating into PLLS. }\end{array}$ \\
\hline & Med11 & $\begin{array}{l}\text { Minimal Sensor Resources in } \\
\text { the EVA suit and PLSS. }\end{array}$ & $\begin{array}{l}\text { - 2.2.3 Must coordinate with industry to acquire or develop COTS sensors to optimize } \\
\text { medical equipment, with a focus on low mass, small volume, and minimum power } \\
\text { requirements. } \\
\text { - } 2.2 .4 \text { Must account for design constraints regarding sensing capabilities of medical } \\
\text { sensors in micro- and zero-gravity environments } \\
\text { - 2,2,5 Must investigate methods for harvesting energy from IVA and EVA environments } \\
\text { to power biosensors. } \\
\text { - 2.2.6 Determine potential alternative uses for the EVA sensors, to enable repurposing } \\
\text { while not being used for medical assessments. } \\
\text { - 2.2.7 Ensure sensor technology reliability in any BLEO environments in which they } \\
\text { may be deployed. } \\
\text { - 2.2.8 Ensure that any wireless transmission of sensor data does not interfere with the } \\
\text { VOR and RCA in the PLSS. }\end{array}$ \\
\hline \multirow[t]{2}{*}{$\begin{array}{l}\text { 2.3 Medical Data Processing } \\
\text { Capabilities }\end{array}$} & \multirow[t]{2}{*}{ Med07 } & IVA astronaut information & $\begin{array}{l}\text { - 2.3.1 Must determine what information can and should be transmitted to the IVA } \\
\text { astronaut, as well as which sensor information, such as ultrasound and accelerometer } \\
\text { data, is sufficient for providing remote guidance. }\end{array}$ \\
\hline & & Autonomous Care & $\begin{array}{l}\text { - } 2.3 .2 \text { Must know sensor input and output ranges before efficient medical algorithms } \\
\text { may be derived. }\end{array}$ \\
\hline
\end{tabular}

Table 2: Research Objectives and Challenges: Biosensing and Bioadvisory Algorithms.

\begin{tabular}{|c|c|c|c|}
\hline Research Stage & Roadmap Gaps & Specific Needs Addressed & Objectives and Challenges \\
\hline 3.1 Heads-Up Display & Med03 & $\begin{array}{l}\text { Situational Awareness } \\
\text { for next-generation EVA } \\
\text { suits to meet goals for a } \\
\text { 24-hour mission in BLEO } \\
\text { environments. }\end{array}$ & $\begin{array}{l}\text { - 3.1.1 Determine MCMs that must be delivered to EVA personnel to enhance their } \\
\text { ability to perceive relevant changes in BLEO environments. } \\
\text { - 3.1.2 Determine graphics that may be used on displays to appropriately convey } \\
\text { mission criticality. } \\
\text { - 3.1.3 Determine the technology for best types of displays and controls options in } \\
\text { the HUD. } \\
\text { - 3.1.4 Render graphics on the HUD in a manner that can adapt to the needs of } \\
\text { color-blind astronauts. } \\
\text { - 3.1.5 Design a user-friendly interface which allows astronauts to personalize their } \\
\text { EVA displays for ease of use and mission-specific information. }\end{array}$ \\
\hline 3.2 Telemedicine Capabilities & Med10 & $\begin{array}{l}\text { Transmission of real-time } \\
\text { MCM Data to IVA personnel. }\end{array}$ & $\begin{array}{l}\text { - 3.2.1 Must integrate framework for autonomous transmission of MCM data to IVA } \\
\text { personnel. } \\
\text { - 3.2.2 Must ensure reliability for EVA BLEO operations. } \\
\text { - 3.2.3 Must research methods for reliable transmission of data during 24-hour EVA } \\
\text { missions in BLEO environments with minimal telemedicine capabilities. }\end{array}$ \\
\hline
\end{tabular}

Table 3: Research Objectives and Challenges: Transmitting and Displaying and Conveying MCMs to EVA and IVA personnel.

corresponding HRP roadmap gaps, the specific needs being addressed, and the objectives and challenges for the corresponding research stage. Table 1 outlines how MCMs must be determined before sensors, devices, and algorithms may be investigated to improve the performance of astronauts. Table 2 outlines challenges in implementing biosensing and bioadvisory devices and algorithms for EVA suit monitoring. Table 3 outlines objectives and challenges related to transmitting and displaying MCM alerts and data to EVA personnel.

\section{Demonstration of MCM Broadcast on OLED}

Most of the interviews conducted by the team occurred during the summer of 2017, and the interview with Dr. Abercomby took place in October 2017. The project is being continued by a senior capstone project at UM EE which is focusing primarily on challenges 3.1.1-3.1.5 from Table 3. The objective of the project is to develop an avenue for rendering real-time MCM data on a HUD using an organic light-emitting diode (OLED) display. Organic LED displays have the potential for integration into the HUD on the helmet itself because they do not need to use a backlight like a conventional LED display, so they may be transparent when no data is being displayed. Therefore, a transparent OLED display on the helmet mask itself is a potential solution for rendering the data in a manner that does not obstruct vision in the EVA suit in the event of device failure.

The focus of the Senior Capstone project is on reducing information fatigue, providing motivational incentives to the astronaut, and designing appealing display aesthetics. Figure 1 illustrates the data flow for an OLED display prototype that we have built based on interview feedback. This initial proof-of-concept utilizes an Adafruit $1.27^{\prime \prime}$ and 1.5" Color OLED Breakout Board [37] with 384 segments and 128 commons output, supporting up to 128RGB x 128 dot matrix display size. This chip is designed for Common Cathode type OLED/ PLED panels, which permits dynamic changing of the MCM data on the display. As shown in Figure 2, three potentiometers were used to represent sensor data; changes in resistance represent changes in MCM data such as $\mathrm{O}_{2}$ and $\mathrm{CO}_{2}$ levels.

The default mode for the display is to show no information, which helps to avoid information fatigue. A microcontroller was used to 


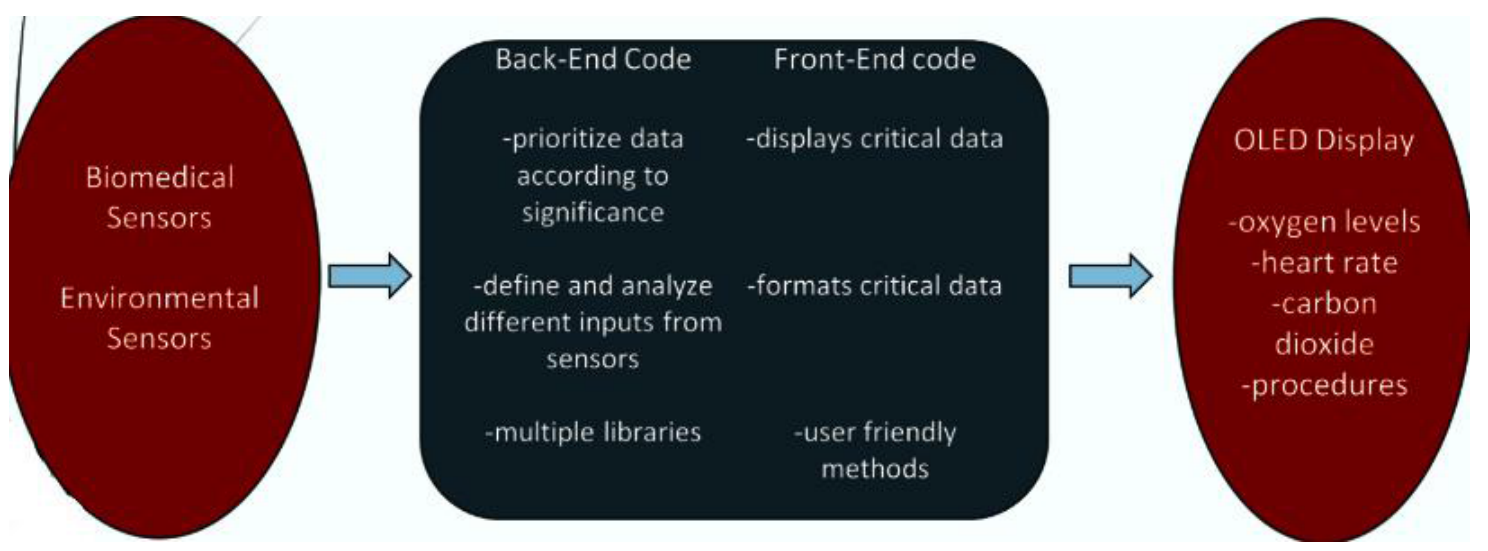

Figure 1: Data Flow Chart for OLED Displays Based on Interview Feedback.

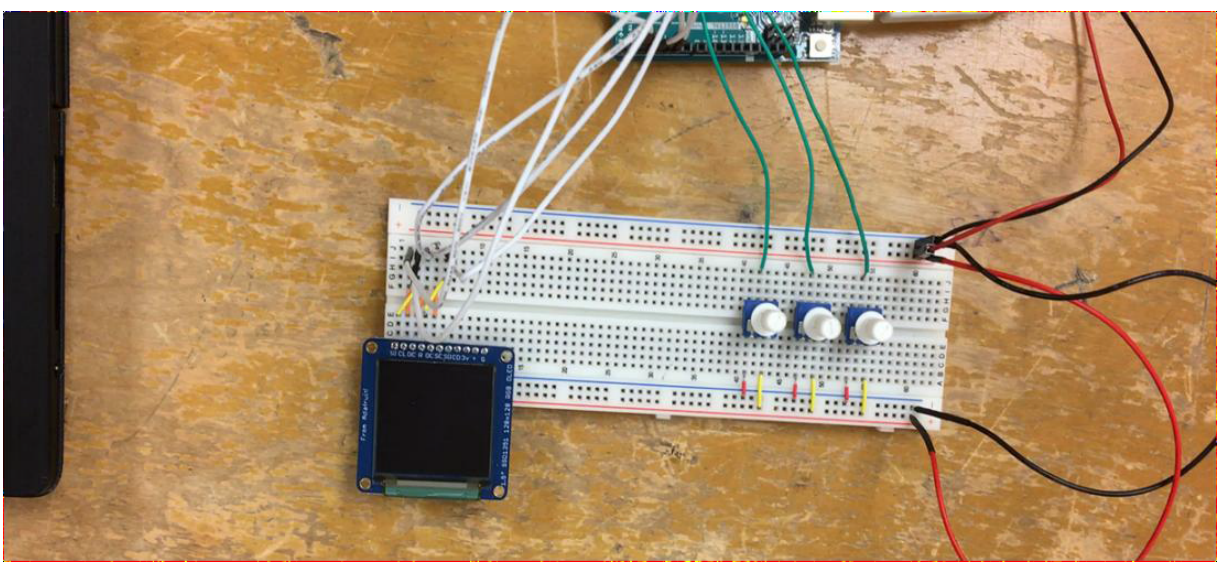

Figure 2: Proof of Concept OLED Display with Potentiometers as Sensors.

relay information to the OLED display. Figure 3 shows the results once one or more thresholds are violated. On the right, the $\mathrm{O}_{2}$ sensor has barely broken the benchmark, so the data is displayed in white. As the potentiometer is changed further, the data dynamically changes to red to indicate a greater sense of danger and indicate to the EVA personnel that the urgency of the situation is increased. The left side image in Figure 3 shows the dynamically changed color for the $\mathrm{O}_{2}$ from white to red, as well as additional MCM data that have exceeded predetermined threshold values ( $\mathrm{CO}_{2}$, "X"). The results of this work are being presented at the Louis Stokes Alliance for Minority Participation Research Conference [38] and NASA Johnson Space Center's EISD Research Day in December 2016 [39], and the Annual Biomedical Research Conference for Minority Students [40] (Figure 1).

Based upon the relevant set of MCMs, we will develop a softwarebased algorithm to detect mission critical levels and to only display that critical data on our OLED, which will be positioned in a corner portion of an astronaut visor. We will develop an algorithm for determining autonomous and augmented medical decision support based upon our feedback from astronauts and researchers. We will determine what data and levels, such as depleted $\mathrm{O}_{2}$ levels or excessive $\mathrm{CO}_{2}$ and humidity levels - which are potentially lethal in low-gravity environments - should be met to interrupt a set of instructions that may be on the OLED display. To test the development of the algorithm, we will use both simulated inputs from a Raspberry Pi controller and manual inputs from potentiometers simulating changes in the MCMs

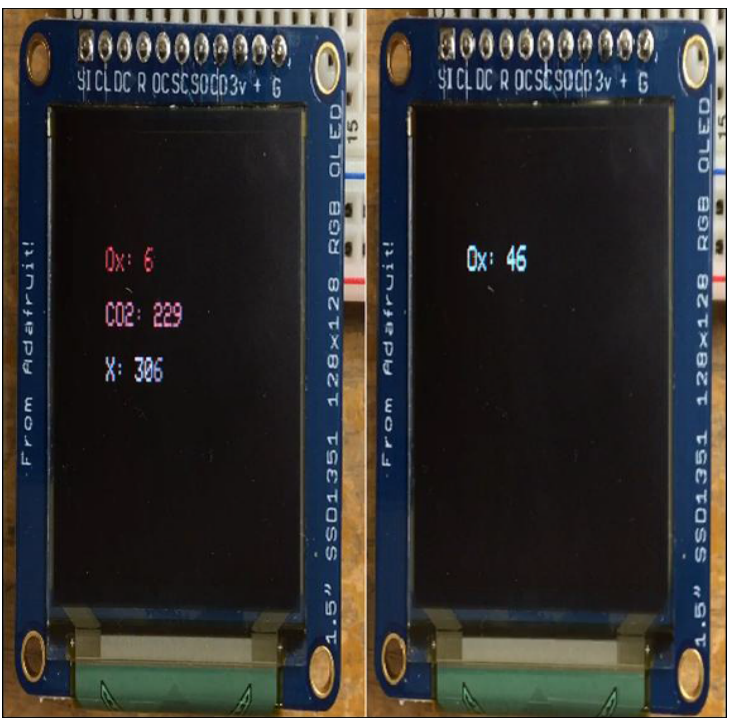

Figure 3: Demonstration of OLED display dynamically displaying MCM data.

to determine the effectiveness of displaying the data in real time. The device representing the IVA personnel should be able to monitor the EVA heart rate, hydration, oxygen levels, carbon dioxide levels, and humidity levels of the suit, but should only display information to the HUD once a MCM requirement is violated (Figures 2 and 3). 
The breadboard was initially configured using an Arduino Uno and Adafruit SSD1351 RGB OLED display, where the breadboard supports the hardware while the MCM variables are simulated. The OLED represented the "mission critical display", and displayed the coded data. The potentiometers simulated sensor data change by alternating resistance. When the MCM has not been violated, the variables are not shown at all. Next, as shown on the right display for Figure 3, when a variable is slightly violated, the variable is shown in white. As the variable becomes exceedingly violated, the value gets darker, indicating that intervention is required by the EVA and IVA personnel.

The testing display configuration was updated using a Raspberry Pi 3 controller for more processing power and configuration options than the Arduino Uno. An MCP3208 Analog-to-Digital converter was integrated since the Raspberry Pi 3 lacks native analog-to-digital conversion. Most importantly, the Python IDE and Graphical User Interface was used to simulate wireless communications of MCM data from the server (representing the IVA astronaut) and the Raspberry Pi client (representing the EVA astronaut). The server provided a method to create a personalized configuration file to simulate the Apollo 16 mission and dictating the setup for the sensor HUD. The program uses that configuration file to build a personalized Heads Up Display wireless communication program containing the desired sensors and sensor parameters representing mission critical measurable. The sensor values were read and displayed according to the configurations file. Finally, using a LoRa (long-range, low power) wireless platform, which uses a low data-rate ( 30 bytes per second) we transmitted the signal using a receiver sensitivity of $-134 \mathrm{dbm}$ and a link budget of $148 \mathrm{~dB}$ over a range of 4.7 miles, which is the furthest distance the Apollo astronauts travel from the Lunar Module on the Moon.

\section{Conclusions}

In this report, we have summarized feedback received from several NASA experts in the field of EVA suit medical monitoring, health and human performance, and BLEO missions. We have also discussed challenges and advances in the use of biosensing in BLEO environments. Based on the above feedback, we have proposed a three-stage research plan for determining appropriate mission-critical measurables, developing biosensing and bioadvisory devices and algorithms, and displaying and conveying MCMs to EVA and IVA personnel, along with corresponding goals and challenges that must be met. By partitioning the tasks in this manner, we aim to enable rapid technological development and advancement in identifying and closing HRP roadmap gaps without changes in one area hindering progress in another. We have outlined the current status of the research infrastructure at UM EE, UM CHSP, and UMMC that would enable a focused research collaboration to meet NASA's mission and objectives.

\section{Acknowledgements}

This work was supported by the National Science Foundation through the Broadband Wireless Access and Applications Center (BWAC) I/UCRC (Award \#1539990). This work was also conducted in collaboration with NASA's Human Research Program through NASA's High Impact/Broad Implementation STEM Education Partnerships Program (EDUCATION01SP16). We would like to acknowledge the NASA and Wyle Laboratories personnel who allowed our research team to interview them and were generous with their time: Dr. Lauren Merkle, Dr. Sara Zwart, Dr. John Charles, Dr. Jennifer Fogarty, Mr. Nathanie Newby, Mr. David Ham, Dr. Daniel Barta, Mr. David Miranda, Dr. Jeffrey Somers, Dr. Andrew Abercromby, Dr. Shane McFarland, Mrs. Amy McCluskey, Dr. Mary Wusk, and Mr. Bill Parsons. We also acknowledge Dr. Igor Dubinsky, Dr. Nicole Stott, and General Charles M. Duke for graciously describing their experiences in astronaut training and EVA missions on the Space Shuttle, International Space Station, and on the Moon.

\section{References}

1. NASA (2011) NASA Human Research Program Specific Objectives

2. NASA Human Research Roadmap Gap EVA 10 (2017) EVA 10: Can knowledge and use of real-time physiological and system parameters during EVA operations improve crew health and performance?

3. NASA Human Research Roadmap Gap Med09 (2017) Med09: We do not have the capability to predict estimated medical risk posture during exploration missions based on current crew health and resources.

4. NASA Human Research Roadmap Gap Med10 (2017) Med10: We do not have the capability to provide computed medical decision support during exploration missions.

5. NASA (2017) International Space Station Medical Monitoring (ISS Medical Monitoring)

6. NASA (2017) ISS Actiwatch Spectrum System

7. PHILIPS (2017) Philips Respironics Actiwatch Spectrum System Brochure.

8. Putro WS, Lestari RE (2015) The scoring quality of astronauts' sleeps using Fuzzy C-Means (FCM) during microgravity effect in the International Space Station (ISS). Scientific Journal of PPI - UKM 2: 3.

9. NASA (2015) EVA Physiology.

10. Gernhardt ML, Jones JA, Scheuring AF, Abercromby JA, Tuxhorn JA, et al. (2009) Risk of Compromised EVA Performance and Crew Health Due to Inadequate EVA Suit Systems. NASA.

11. Scheuring RA, Jones JA, Polk JD, Gillis DB, Schmid JF, et al. (2007) The Apollo Medical Operations Project: recommendations to improve crew health and performance for future exploration missions and lunar surface operations. TM-2007-214755. NASA Johnson Space Center, Houston.

12. Jones JA, Ansari R, Das H, Dewitt JK, Gernhardt ML, et al. (2006) Medica issues for extravehicular activity (EVA). Presentation at the National Space Biomedical Research Institute Retreat. Houston, Feb 27 - Mar 1, 2006.

13. Fischer E, Haines RF, Price TA (1980) Cognitive Issues in Heads-Up Displays NASA Technical Paper 1711.

14. Fischer E (1979) The Role of Cognitive Switching in Head-Up Displays. NASA CR-3137.

15. Naish JM, Miller DL (1980) An Experimental Evaluation of Head-Up Display Formats. NASA TP-1550.

16. Miranda DJ, Villorin A, VanNorma M, McDonough M (2016) IDEAS To Enhance Operations on Earth and in Space.

17. Villorin A (2016) IDEAS: Integrated Display and Environmental Awareness System. NASA's Game Changing Technology Industry Day presentation.

18. Barta D (2016) EVA Life Support Technologies. NASA's Game Changing Technology Industry Day presentation.

19. Smith SM, Zwart SR, Heer M (2014) Human Adaptation to Spaceflight: The Role of Nutrition. NASA.

20. Barta DJ, Ewert MK (2009) Development of Life Support System Technologies for Human Lunar Missions. NASA.

21. NASA (2008) International Space Station Environmental Control and Life Support System. NASA FS-2008-05-83-MSFC.

22. Norcross JR, McFarland SM (2016) Development of an Objective Space Suit Mobility Performance Metric Using Metabolic Cost and Functional Tasks. NASA.

23. NASA HRP Task (2017) Biosensors for Exploration Medical System.

24. Miller J (2016) Inventing the Apollo Spaceflight Biomedical Sensors. Smithsonian National Air and Space Museum.

25. Pacela, AF (1966) Impedance pneumography-A survey of instrumentation techniques. Journal of Medical and Biological Engineering 4:1-15.

26. Lee SMC, Ellerby G, Scott P, Stroud L (2009) Biosensors for EVA: Muscle Oxygen and $\mathrm{pH}$ During Walking, Running and Simulated Reduced Gravity. NASA Technical Reports Server.

27. Blaauw D, Sylvester D, Dutta P. "IoT design space challenges: Circuits and 
Citation: Morrison M, Rogers R, Singh K, Harper B, Sanchez F, et al. (2017) Research Gaps and Opportunities in Sensor-Based Medical Exploration Capabilities in Extravehicular Astronaut Suits. J Biosens Bioelectron 8: 248. doi: 10.4172/2155-6210.1000248

Page 9 of 9

systems. 2014 Symposium on VLSI Technology (VLSI-Technology): Digest of Technical Papers, Honolulu.

28. Ralston JD, Woodard J, Cieslak M, Asturias A, Meiring W, et al. (2017) A Candidate Neuromechanical Biomarker and Dosimeter for Monitoring Cumulative Head Impact Trauma", IEEE EMBS International Conference on Biomedical \& Health Informatics (BHI), Orlando.

29. Jules K, Lin P (2000) ISS Microgravity Environment Monitoring System (MEMS). Part 1; System Design" NASA Technical Reports Server.

30. Mallis MM, DeRoshia CW (2005) Circadian Rhythms, Sleep, and Performance in Space. Aviation, Space, and Environmental Medicine. 76: B94-107.

31. Ralston JD, Meiring W, Cieslak M, Asturias A, Grafton ST (2017) Wearable Sensors for Head Impact Dosimetry. Proceedings of IEEE Sensors 2017, Oct 30 - Nov 1. Glasgow, Scotland.

32. Morrison M, Daigle JN, Ralston J (2015) A Biosensing Approach for Detecting and Managing Head Injuries in American Football. J Biosens Bioelectron 6:189.

33. Tissier J, Latrach M (2017) Broadband rectenna for ambient RF energy harvesting applications. 2017 XXXIInd General Assembly and Scientific
Symposium of the International Union of Radio Science (URSI GASS), Montreal, QC, Canada, 2017, pp. 1-3.

34. Kauer M, Bellanger M (2017) Development of an Indoor Photovoltaic Energy Harvesting Module for Autonomous Sensors in Building Air Quality Applications. IEEE Internet of Things Journal 99: 1-1.

35. Kovo Y (2017) Technology Opportunity: Compliant Electrode and Composite Materials for Piezoelectric Wind and Mechanical Energy Conversions.

36. Chen B (2015) Compliant electrode and composite material for piezoelectric wind and mechanical energy conversions. U.S. Patent 9024510 B1.

37. Solomon Systech (2009) OLED/PLED Sigment Common Driver with Controller.

38. Rodgers R, Singh K, Williams T, Morrison M (2016) Exploring Sensor Solutions for NASA's Medical Capability", Mississippi Louis Stokes Minority Participation IMAGE Conference.

39. Rodgers R, Singh K, Williams O, Morrison M (2016) Integration of Biomedical Sensors for Heads Up Display Unit for EVA Health Monitoring. NASA JSC EISD Research Day.

40. Rogers R, Morrison M (2016) Exploring NASA's Medical Sensor Capability Annual Biomedical Research Conference for Minority Students. 